\title{
Spike Rate Estimation Using Bayesian Adaptive Kernel Smoother (BAKS) and Its Application to Brain Machine Interfaces
}

\author{
Nur Ahmadi ${ }^{1,2}$, Student Member, IEEE, Timothy G. Constandinou ${ }^{1,2}$, Senior Member, IEEE, and \\ Christos-Savvas Bouganis ${ }^{1}$, Member, IEEE
}

\begin{abstract}
Brain Machine Interfaces (BMIs) mostly utilise spike rate as an input feature for decoding a desired motor output as it conveys a useful measure to the underlying neuronal activity. The spike rate is typically estimated by a using nonoverlap binning method that yields a coarse estimate. There exist several methods that can produce a smooth estimate which could potentially improve the decoding performance. However, these methods are relatively computationally heavy for real-time BMIs. To address this issue, we propose a new method for estimating spike rate that is able to yield a smooth estimate and also amenable to real-time BMIs. The proposed method, referred to as Bayesian adaptive kernel smoother (BAKS), employs kernel smoothing technique that considers the bandwidth as a random variable with prior distribution which is adaptively updated through a Bayesian framework. With appropriate selection of prior distribution and kernel function, an analytical expression can be achieved for the kernel bandwidth. We apply BAKS and evaluate its impact on offline BMI decoding performance using Kalman filter. The results show that overlap BAKS improved the decoding performance up to $3.33 \%$ and $12.93 \%$ compared to overlap and non-overlap binning methods, respectively, depending on the window size. This suggests the feasibility and the potential use of BAKS method for real-time BMIs.
\end{abstract}

\section{INTRODUCTION}

According to rate coding theory, information about behavioral tasks are contained in the neuronal firing rate, that is the rate or frequency at which a neuron fires spikes. Firing rate (also known as spike rate) has been the most commonly used input feature for brain-machine interface (BMI), a system that enables paralyzed patients control external devices through their brain activity. In this type of BMI system, the spike rate is commonly estimated using simple binning method by counting the number of spikes within predefined non-overlap bins [1]. Despite simple and fast, this binning method results in a coarse or noisy estimate, which may affect the decoding performance. By utilising a smooth estimate of spike rate, the decoding performance could potentially be improved. There exist several methods that can yield a smooth estimate of spike rate by employing automatic selection of optimal smoothing parameter, such

\footnotetext{
*Nur Ahmadi is supported by the graduate scholarship awarded by Indonesia Endowment Fund for Education (LPDP), Republic of Indonesia.

${ }^{1}$ Nur Ahmadi, Timothy G. Constandinou, and Christos-Savvas Bouganis are with the Department of Electrical and Electronic Engineering, Imperial College London, SW7 2BT, UK. Email: \{n.ahmadi16, t.constandinou, christos-savvas.bouganis\}@imperial.ac.uk

${ }^{2}$ Nur Ahmadi and Timothy G. Constandinou are additionally with the Centre for Bio-Inspired Technology, Institute of Biomedical Engineering, Imperial College London, SW7 2AZ, UK
}

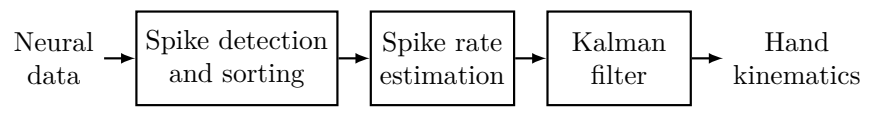

Fig. 1. Block diagram of brain-machine interface

as fixed and variable kernel smoothing techniques [2] and Bayesian adaptive regression splines (BARS) [3]. However, these methods require relatively long time computation time with respect to real-time constraint of BMI applications. This might be one of the reasons why there lacks of adoption of smoothing techniques for spike rate estimation in BMI setting. In light of this, it is desirable to have a method that can produce a smooth estimate of spike rate while keeping the computation time within the real-time constraint.

In this paper, we propose a new method for estimating the spike rate, which is adaptive, automatic, and amenable to real-time BMI. The proposed method employs kernel smoothing technique with the bandwidth treated as random variable and adaptively and automatically determined under Bayesian framework. By selecting appropriate prior distribution of bandwidth and kernel function, an analytical expression of posterior bandwidth can be obtained which reduces the computational complexity. The proposed method is referred to as Bayesian adaptive kernel smoother (BAKS). We apply the BAKS as input feature extractor for decoding hand kinematics from neural data recorded from motor cortex (M1) area of non-human primate (NHP). We investigate and compare the decoding performance impacted by the BAKS and binning methods under Kalman filter decoder. We also compare the estimated computation time of both BAKS and binning methods.

\section{METHODS}

BMI system used in this work is illustrated in Fig. 1. The preprocessing steps include filtering raw neural data, detecting spikes and sorting them into putative single cells. Spike rate estimation is performed using BAKS or binning methods before being fed to a decoding algorithm. Kalman filter is chosen as it has been widely used as BMI decoder and shown to yield a good decoding performance [4]-[6].

\section{A. Bayesian Adaptive Kernel Smoother (BAKS)}

BAKS estimates spike rate, $\hat{\lambda}(t)$, by employing a kernel smoothing technique which convolves a spike train $\rho(t)$ with 
a kernel function $K_{h(t)}(t)$,

$$
\hat{\lambda}(t)=\int_{-\infty}^{\infty} K_{h(t)}(\tau) \rho(t-\tau) d \tau
$$

where $h(t)$ denotes adaptive (instead of fixed) bandwidth parameter. The spike train is mathematically expressed as

$$
\rho(t)=\sum_{i=1}^{n} \delta\left(t-t_{i}\right)
$$

where $t_{i}$ represents the spike time, $n$ is the number of spikes, and $\delta(t)$ is Diract function. By substituting Eq. (2) into Eq. (1), we can obtain,

$$
\hat{\lambda}(t)=\sum_{i=1}^{n} K_{h(t)}\left(t-t_{i}\right)
$$

We propose a Gaussian kernel function and Gamma prior distribution on the precision parameter $(\sigma(t))$, where $\sigma(t)=1 / h(t)^{2}$. Due to conjugate prior relationship, this combination leads to analytical expression of the posterior distribution. The Gamma prior distribution is given by

$$
\pi(\sigma(t))=\frac{\sigma(t)^{\alpha-1}}{\Gamma(\alpha) \beta^{\alpha}} \exp \left\{-\frac{\sigma(t)}{\beta}\right\}, \quad \sigma>0
$$

where $\alpha>0$ is the shape parameter, $\beta>0$ is the scale parameter, and $\Gamma(\alpha)$ is Gamma function. By the change-ofvariable formula and transformation technique, Eq. (4) can be expressed as:

$$
\pi(h(t))=\frac{2 h(t)^{-2 \alpha-1}}{\Gamma(\alpha) \beta^{\alpha}} \exp \left\{-\frac{1}{\beta h(t)^{2}}\right\}
$$

Using Bayes' theorem, the posterior distribution of kernel bandwidth, $\pi(h(t) \mid \rho(t))$, can be computed by

$$
\pi(h(t) \mid \rho(t))=\frac{\hat{f}(\rho(t) \mid h(t)) \pi(h(t))}{\int \hat{f}(\rho(t) \mid h(t)) \pi(h(t)) d h(t)}
$$

where $\hat{f}(\rho(t) \mid h(t))$ represents an approximation of likelihood function based on Gaussian kernel, which is formulated as

$$
\hat{f}(\rho(t) \mid h(t))=\frac{1}{n} \sum_{i=1}^{n} \frac{1}{\sqrt{2 \pi} h(t)} \exp \left\{-\frac{\left(t-t_{i}\right)^{2}}{2 h(t)^{2}}\right\}
$$

By substituting Eq. (5) and Eq. (7) into Eq. (6), we can obtain analytical expression as follows

$$
\pi(h(t) \mid \rho(t))=\frac{\sum_{i=1}^{n} h(t)^{-2 \alpha-2} \exp \left\{-\frac{1}{h(t)^{2}}\left[\frac{\left(t-t_{i}\right)^{2}}{2}+\frac{1}{\beta}\right]\right\}}{\frac{1}{2} \Gamma\left(\alpha+\frac{1}{2}\right) \sum_{i=1}^{n}\left[\frac{\left(t-t_{i}\right)^{2}}{2}+\frac{1}{\beta}\right]^{\left(-\alpha-\frac{1}{2}\right)}}
$$

Under squared error loss function, the adaptive bandwidth estimate can be computed by

$$
\hat{h}(t)=\frac{\Gamma(\alpha) \sum_{i=1}^{n}\left[\frac{\left(t-t_{i}\right)^{2}}{2}+\frac{1}{\beta}\right]^{-\alpha}}{\Gamma\left(\alpha+\frac{1}{2}\right) \sum_{i=1}^{n}\left[\frac{\left(t-t_{i}\right)^{2}}{2}+\frac{1}{\beta}\right]^{-\alpha-\frac{1}{2}}}
$$

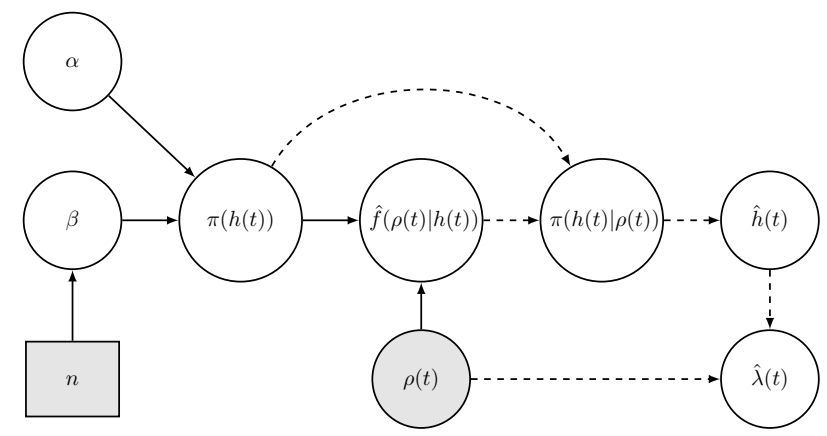

Fig. 2. Graphical representation of BAKS

The adaptive bandwidth in Eq. (9) is then used for spike rate estimation in Eq. (3). The complete derivation of bandwidth posterior distribution and the adaptive bandwidth estimate are provided in [7]. The graphical illustration of BAKS method is depicted in Fig. 2. The circular blocks denote continuous variables, and rectangle blocks denote discrete variables. Shaded blocks represent observed variables, whereas white blocks represent hidden variables. Solid and dashed arrow indicate Bayesian modeling and inference phase, respectively.

Parameter $\alpha$ and $\beta$ in the BAKS method are set to 4 and $n^{4 / 5}$, where $n$ represents the number of spikes. This parameter setting follows the work described in [7].

\section{B. Kalman Filter}

Kalman filter is a popular decoding algorithm and has been applied in many BMI research [6], [8]-[10]. Kalman filter assumes that neural activity (i.e. spike rate) is linearly related to hand kinematics plus Gaussian noise, mathematically expressed as

$$
\mathbf{z}_{k}=\mathbf{H}_{k} \mathbf{x}_{k}+\mathbf{q}_{k}, \quad \mathbf{q}_{k} \sim N\left(0, \mathbf{Q}_{k}\right)
$$

where $\mathbf{z}_{k}$ is spike rates with size of $D \times 1$ calculated every discrete time point $k$ with interval $\Delta t$ (4 ms in this study), and $D$ is the number of cells. Hand kinematics used in this work include horizontal and vertical position, velocity, and acceleration $\left(\left[x, y, v_{x}, v_{y}, a_{x}, a_{y}\right]_{k}^{T}\right)$. $\mathbf{H}_{k}$ is $D \times 6$ transformation matrix, $\mathbf{q}_{k}$ is neural noise vector and $\mathbf{Q}_{k}$ is $D \times D$ neural noise covariance matrix.

Hand kinematics dynamically evolves from previous state according to linear transformation with Gaussian noise formulated as

$$
\mathbf{x}_{k+1}=\mathbf{A}_{k} \mathbf{x}_{k}+\mathbf{w}_{k}, \quad \mathbf{w}_{k} \sim N\left(0, \mathbf{W}_{k}\right)
$$

where $\mathbf{A}_{k}$ denotes $6 \times 6$ transition matrix between kinematic states and $\mathbf{w}_{k}$ is process noise vector. Parameter $\mathbf{A}_{k}, \mathbf{W}_{k}, \mathbf{H}_{k}$, and $\mathbf{Q}_{k}$ are estimated using a least square regression on the training data as described in [11].

\section{Neural Dataset and Behavioural Task}

In this study, we use three datasets associated with three different sessions each with duration of 13, 14, and 15 minutes and number of cells of 218, 221, and 221, respectively. These datasets correspond to neural data from motor 

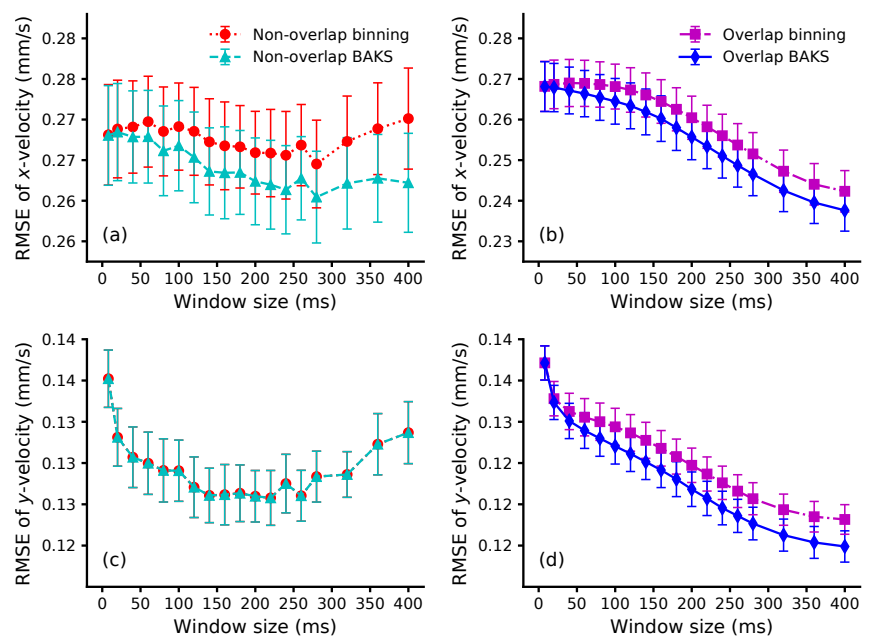

Fig. 3. Comparison of RMSE of $x$ - and $y$-velocity predicted by BAKS and binning methods. Error bars indicate $95 \%$ confidence interval.

cortex (M1) of monkey I recorded by Sabes Lab [12]. The neural data were acquired using 96-channel Utah array while the monkey performing self-paced reaching tasks without gaps or pre-movement delay intervals. Finger position in $\mathrm{x}-\mathrm{y}$ coordinates was sampled at every $4 \mathrm{~ms}$. Velocity and acceleration were computed from first and second derivative of position. The concatenation of position, velocity, and acceleration are referred to as hand kinematics. Neural data acquisition setting, spike detection and sorting are described in [13].

\section{Performance Metrics}

To assess the decoding performance impacted by the BAKS and binning methods, We use two common metrics, which are root mean squared error (RMSE) and coefficient of correlation (CC). These metrics are respectively computed using the following formula:

$$
\begin{gathered}
\mathrm{RMSE}=\left[\left(\sum_{i=1}^{N}\left(\hat{v}_{i}-v_{i}\right)^{2}\right) / N\right]^{\frac{1}{2}} \\
\mathrm{CC}=\frac{\sum_{i=1}^{N}\left(v_{i}-\bar{v}\right)\left(\hat{v}_{i}-\overline{\hat{v}}_{i}\right)}{\left[\sum_{i=1}^{N}\left(v_{i}-\bar{v}\right)^{2} \sum_{i=1}^{N}\left(\hat{v}_{i}-\overline{\hat{v}}_{i}\right)^{2}\right]^{\frac{1}{2}}}
\end{gathered}
$$

where $\hat{v}_{i}$ and $v_{i}$ represents predicted and true velocity at time step $i$, respectively and $N$ is the total number of samples.

\section{RESUlTS}

We divided each dataset into 10 chunks of data and performed 10-fold cross validation. The 8 chunks of data were used for training the Kalman filter, and the remaining chunks of data were used for validation and testing. Training was done to find the optimal parameter of Kalman filter while validation was performed to find the optimal lag between the hand kinematics and spike rate by minimizing RMSE between predicted and true kinematics. All the program codes were written in Python 2.7 and run on Linux OS 64-bit machine with Intel Xeon CPU E5-2680 v3@2.50 GHz.
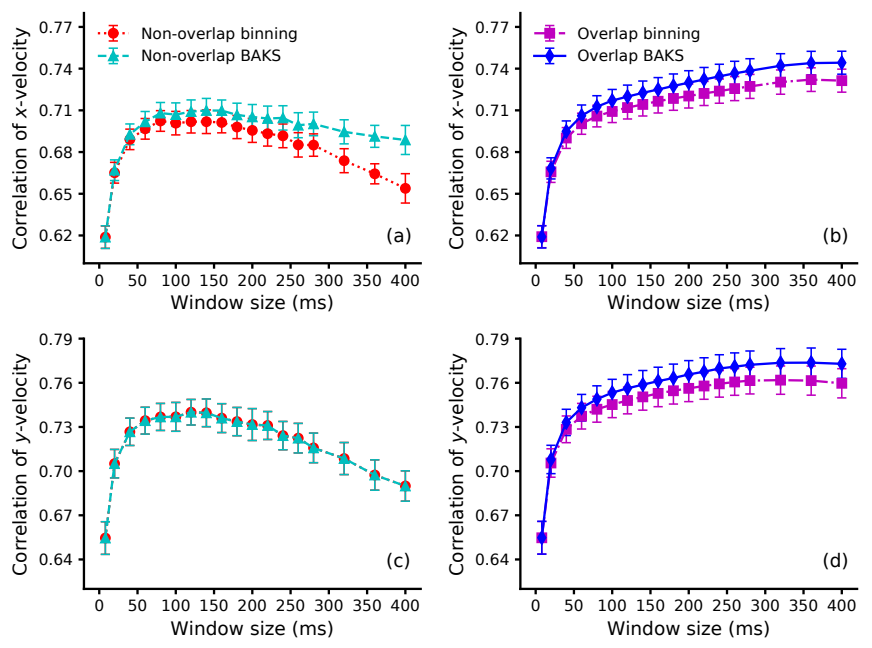

Fig. 4. Comparison of correlation of $x$ - and $y$-velocity predicted by BAKS and binning methods. Error bars indicate $95 \%$ confidence interval.

We tested the trained Kalman filter using spike rates estimated from both BAKS and binning methods, each with two different schemes: non-overlap and overlap. We compared the decoding performance impacted by the spike rates from these schemes under different window size $\{8,20,40,60,80, \cdots, 260,280,320,360,400\} \mathrm{ms}$. The prediction was computed at $4 \mathrm{~ms}$ timescale. The Kalman filter predicts kinematics variables (position, velocity, and acceleration) in $x-y$ Cartesian coordinates. However, in this paper, only the decoding performance of $x$ - and $y$-velocity are shown.

The summary of performance comparison between the BAKS and binning methods from three datasets are illustrated in Fig. 3 and Fig. 4, respectively. In all cases, the BAKS method consistently outperformed the binning method for both RMSE and CC metrics as shown in Fig. 3 and 4. In the case of non-overlapping scheme, the optimal window size is between $120-280 \mathrm{~ms}$; while for the overlapping scheme, the optimal size is between $360-400 \mathrm{~ms}$. The improvement made by the BAKS increases as the window size increases. The maximum average performance improvement by the non-overlap BAKS over the non-overlap binning is $3.33 \%$ (2.24\%) for RMSE (CC). The maximum average performance improvement by the overlap BAKS over the overlap binning is $2.36 \%(1.75 \%)$ for RMSE (CC). When comparing the overlap BAKS with non-overlap binning, we can obtain maximum average performance improvement of $12.89 \%$ (12.93\%) for RMSE (CC).

The computational complexity reflects the execution time. To give an indication of the computational complexity of the BAKS compared to the binning method, we computed the time needed (i.e. elapsed time) by each method to produce the spike rate estimate given a single spike train under different window size. We used Python function time() to compute elapsed time of BAKS and binning method through multiple iterations. We computed the average elapsed time of each method and its $95 \%$ confidence interval which is 


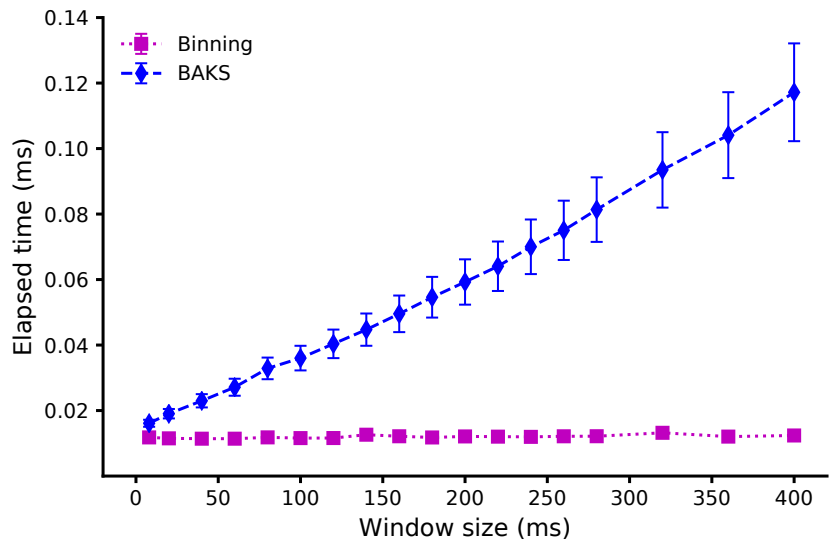

Fig. 5. Comparison of elapsed time between BAKS and binning methods. Error bars indicate $95 \%$ confidence interval.

illustrated in Fig. 5. As the window size increases, the binning method on average takes relatively constant elapsed time, that is $11.8 \pm 0.7 \mu s(12.4 \pm 0.7 \mu s)$ for $8 \mathrm{~ms}(400 \mathrm{~ms})$ window size. On the other hand, the elapsed time of the BAKS method increases as the window size increases. The BAKS takes an average of $16.1 \pm 1.1 \mu \mathrm{s}(117.2 \pm 14.9 \mu \mathrm{s})$ for $8 \mathrm{~ms}$ (400 ms) window size. This corresponds to $1.37-9.46$ times slower than that of the binning method. Even though BAKS method requires larger elapsed time, this value is still amenable to real-time application.

\section{Discussion}

This study demonstrates that employing a smoothing technique for spike rate estimation is able to improve the decoding performance. This improvement is consistently observed for different window size. This smoothing may act as 'denoising' input signal for the decoder to provide better regularization. Performing binning method in an overlapping fashion can also be considered as simple smoothing technique, which also provides performance improvement over the non-overlap counterpart. Our results are in agreement with the previous study [14] showing that a smooth estimate of spike rate can provide an improvement over simple binning method.

Our proposed method, BAKS, allows for a smooth estimation of spike rate in finer timescale. Some studies suggest that faster control rate and feedback rate, which corresponds to finer time scale, may improve the decoding performance [5], [15]. The BAKS method computed in an overlapping fashion provides significant improvement compared to nonoverlapping binning. This improvement comes at the expense of increased execution or elapsed time. In this study, we have not imposed any optimization on our implementation codes. We may reduce the elapsed time by using optimization technique. This work only compares the performance under offline BMI decoding which does not directly translate into similar improvement in the online BMI setting. In addition, we only use Kalman filter decoding algorithm for performance benchmark. A more thorough assessment of the impact of BAKS to BMI decoding performance under different decoding algorithm will be performed in the future work. In addition, it is also of interest to apply BAKS and evaluate its impact to decoding performance in online BMI setting.

\section{ACKNOWLEDGEMENT}

We thank J. E. O'Doherty and P. N. Sabes for making their data publicly available.

\section{REFERENCES}

[1] M. M. Shanechi, "Brain-machine interface control algorithms," IEEE Transactions on Neural Systems and Rehabilitation Engineering, vol. 25, no. 10, pp. 1725-1734, 2017.

[2] H. Shimazaki and S. Shinomoto, "Kernel bandwidth optimization in spike rate estimation," Journal of computational neuroscience, vol. 29, no. 1-2, pp. 171-182, 2010.

[3] I. DiMatteo, C. R. Genovese, and R. E. Kass, "Bayesian curve-fitting with free-knot splines," Biometrika, pp. 1055-1071, 2001.

[4] W. Wu, Y. Gao, E. Bienenstock, J. P. Donoghue, and M. J. Black, "Bayesian population decoding of motor cortical activity using a kalman filter," Neural computation, vol. 18, no. 1, pp. 80-118, 2006.

[5] J. P. Cunningham, P. Nuyujukian, V. Gilja, C. A. Chestek, S. I. Ryu, and K. V. Shenoy, "A closed-loop human simulator for investigating the role of feedback control in brain-machine interfaces," Journal of neurophysiology, vol. 105, no. 4, pp. 1932-1949, 2011.

[6] S.-P. Kim, J. D. Simeral, L. R. Hochberg, J. P. Donoghue, and M. J. Black, "Neural control of computer cursor velocity by decoding motor cortical spiking activity in humans with tetraplegia," Journal of neural engineering, vol. 5, no. 4, p. 455, 2008.

[7] N. Ahmadi, T. G. Constandinou, and C.-S. Bouganis, "Estimation of neuronal firing rate using Bayesian adaptive kernel smoother (BAKS)," bioRxiv, p. 204818, 2017.

[8] W. Wu, M. J. Black, Y. Gao, M. Serruya, A. Shaikhouni, J. Donoghue, and E. Bienenstock, "Neural decoding of cursor motion using a kalman filter," in Advances in neural information processing systems, 2003, pp. 133-140.

[9] F. R. Willett, B. A. Murphy, D. R. Young, W. D. Memberg, C. H. Blabe, C. Pandarinath, B. Franco, J. Saab, B. L. Walter, J. A. Sweet et al., "A comparison of intention estimation methods for decoder calibration in intracortical brain-computer interfaces," IEEE Transactions on Biomedical Engineering, 2017.

[10] Z. Irwin, K. Schroeder, P. Vu, A. Bullard, D. Tat, C. Nu, A. Vaskov, S. Nason, D. Thompson, J. Bentley et al., "Neural control of finger movement via intracortical brain-machine interface," Journal of neural engineering, vol. 14, no. 6, p. 066004, 2017.

[11] W. Wu, M. Black, Y. Gao, E. Bienenstock, M. Serruya, and J. Donoghue, "Inferring hand motion from multi-cell recordings in motor cortex using a kalman filter," in SAB02-workshop on motor control in humans and robots: On the interplay of real brains and artificial devices, 2002, pp. 66-73.

[12] J. E. O'doherty, M. M. B. Cardoso, J. G. Makin, and P. N. Sabes, "Nonhuman primate reaching with multichannel sensorimotor cortex electrophysiology," 2017, doi:10.5281/zenodo.583331.

[13] J. G. Makin, J. O’Doherty, M. M. Cardoso, and P. N. Sabes, "Superior arm-movement decoding from cortex with a new, unsupervisedlearning algorithm," Journal of neural engineering, 2017.

[14] J. P. Cunningham, V. Gilja, S. I. Ryu, and K. V. Shenoy, "Methods for estimating neural firing rates, and their application to brain-machine interfaces," Neural Networks, vol. 22, no. 9, pp. 1235-1246, 2009.

[15] M. M. Shanechi, A. L. Orsborn, H. G. Moorman, S. Gowda, S. Dangi, and J. M. Carmena, "Rapid control and feedback rates enhance neuroprosthetic control," Nature communications, vol. 8, p. 13825 2017. 